\title{
On flux terms in volume averaging
}

\author{
Shigan $\mathrm{Chu}^{\mathrm{a}}$, Andrea Prosperetti ${ }^{\mathrm{a}, \mathrm{b}, 1}$ \\ ${ }^{a}$ Department of Mechanical Engineering, Johns Hopkins University, Baltimore MD \\ 21218 USA \\ ${ }^{b}$ Department of Applied Sciences and Burgerscentrum, University of Twente, Enschede, \\ The Netherlands
}

\begin{abstract}
This note examines the modelling of non-convective fluxes (e.g. stress, heat flux and others) as they appear in the general, unclosed form of the volumeaveraged equations of multiphase flows. By appealing to the difference between slowly and rapidly varying quantities, it is shown that the natural closure of these terms leads to the use of a single, slowly-varying combined average flux, common to both phases, plus rapidly-varying local contributions for each phase. The result is general and only rests on the hypothesis that the spatial variation of the combined average flux is adequately described by a linear function of position within the averaging volume. No further hypotheses on the nature of the flow (e.g., about specific flow regimes) prove necessary. The result agrees with earlier ones obtained by ensemble averaging, is illustrated with the example of disperse flows and discussed in the light of some earlier and current literature. A very concise derivation of the general averaged balance equation is also given.
\end{abstract}

Keywords: volume averaging, separation of scales 
is a crucial point as, in the absence of physical transparency, it becomes harder to develop physically relevant closures.

The specific aspect on which we focus in this note is the modelling of nonconvective fluxes (e.g, stress, heat flux, diffusive flux) in the averaged balance equations. The basis of the approach is the recognition that, in a spatially non-homogeneous flow, simultaneously with the slow spatial dependence of the macroscopic averaged quantities, there is a faster, local spatial dependence of the microscopic fields. A procedure which takes into consideration this multi-scale nature of the actual situation results in a physically transparent form of the averaged equations (section 3) which, in turn, helps to close the equations as we show with the example of a disperse flow in section 4. Section 5 provides a discussion of the results in the context of the existing literature. Section 2 contains a very synthetic ("efficient") derivation of the volume-averaged equations in the standard form in which they are usually presented.

\section{The general averaged balance law}

We consider for simplicity a general system consisting of two phases, denoted by indices 1 and 2, although extension of the procedure to the more general case of three or more phases is straightforward.

We attach to each point $\mathbf{x}$ in space an averaging volume $V(\mathbf{x})$ of fixed shape and orientation; $V_{j}(\mathbf{x}, t)$, with $j=1$ or 2 , denotes the (generally timedependent) part of $V$ occupied by the $j$-th phase so that $V=V_{1}+V_{2}$. The surface $S$ of $V$ is also decomposed in the same way, $S=S_{1}+S_{2}$, with $S_{j}$ the portion of $S$ occupied by the $j$-th phase (see figure 1). Inside $V$ the two phases are separated by an interface $S_{i}$, possibly consisting of disjoint parts as, e.g., in the case of droplets suspended in a continuous phase. It is important to keep in mind that the volume occupied by the $j$-th phase inside the averaging volume is bounded by $S_{j}+S_{i}$ which, therefore, is a closed surface.

The volume average of a generic quantity $q_{j}$ of arbitrary tensorial order belonging to the $j$-th phase is defined as

$$
\left\langle q_{j}\right\rangle(\mathbf{x}, t)=\frac{1}{V_{j}(\mathbf{x}, t)} \int_{V_{j}(\mathbf{x}, t)} q_{j}(\boldsymbol{\xi}, t) d^{3} \xi,
$$

where $\mathbf{x}$ is the position of the averaging volume. Upon using the (generalized) 


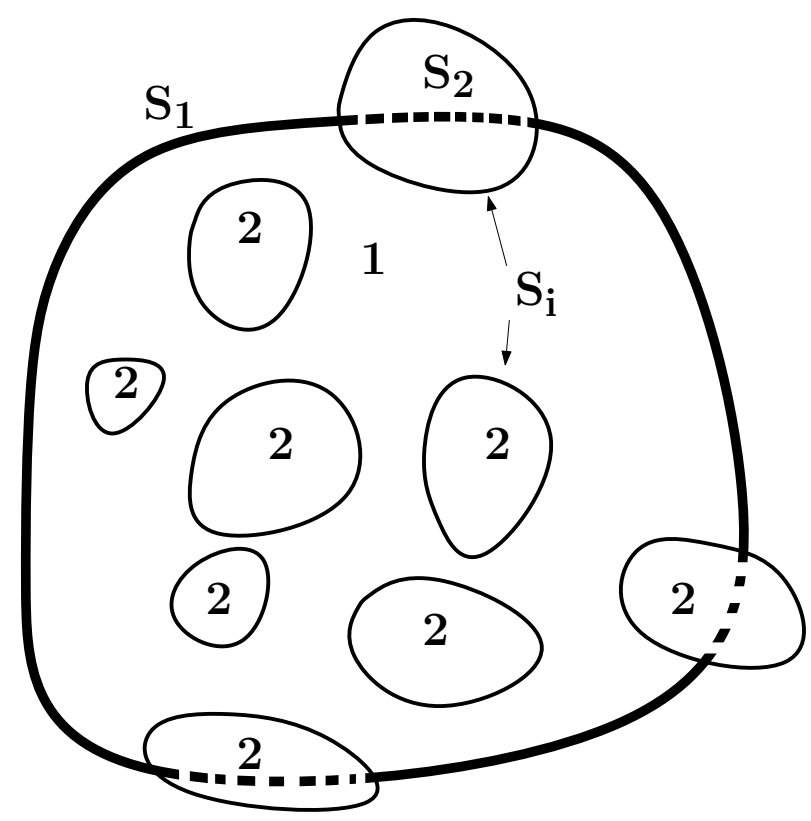

Figure 1: Averaging volume in a two-phase system. The surface of the averaging volume, $S=S_{1}+S_{2}$, consists of a portion $S_{1}$ in contact with phase 1 (continuous thick line) and a portion $S_{2}$ in contact with phase 2 (dashed thick line). Inside the averaging volume the two phases are separated by an interface $S_{i}$ (thin lines) which may consist of disjoint parts as in this figure.

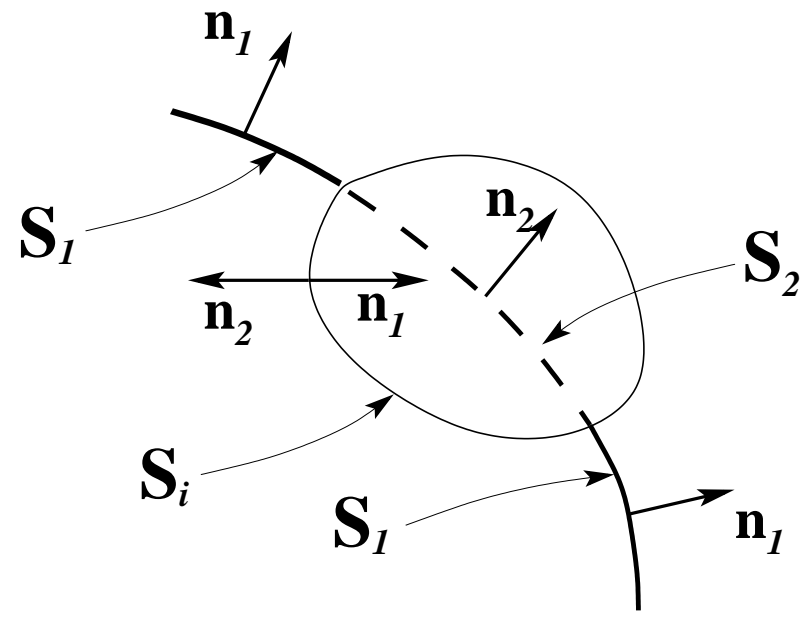

Figure 2: The unit normals are defined to be directed out of the corresponding phase. 
49

54

$$
\frac{\partial}{\partial t}\left(V_{j}\left\langle q_{j}\right\rangle\right)=\int_{V_{j}} \frac{\partial q_{j}}{\partial t} d^{3} \xi+\oint_{S_{j}+S_{i}} q_{j} \mathbf{v} \cdot \mathbf{n}_{j} d S,
$$

where $\mathbf{v}$ is the local velocity of the integration surface and $\mathbf{n}_{j}$ is the unit normal directed out of the $j$-th phase (figure 2). Since $V$ is fixed, $\mathbf{v}=0$ on $S_{j}$ and we are left with

$$
\frac{\partial}{\partial t}\left(V_{j}\left\langle q_{j}\right\rangle\right)=\int_{V_{j}} \frac{\partial q_{j}}{\partial t} d^{3} \xi+\int_{S_{i}} q_{j} \mathbf{v}_{i} \cdot \mathbf{n}_{j} d S_{i},
$$

where $\mathbf{v}_{i}$ is the velocity of the interface contained within $V$. We assume that the quantity $q_{j}$ satisfies a general balance equation of the form

$$
\frac{\partial q_{j}}{\partial t}=-\boldsymbol{\nabla} \cdot\left(\mathbf{u}_{j} q_{j}\right)+\boldsymbol{\nabla} \cdot \boldsymbol{\phi}_{j}+\theta_{j},
$$

where $\mathbf{u}_{j}$ is the $j$-th phase velocity, $\boldsymbol{\phi}_{j}$ the non-convective flux of $q_{j}$ and $\theta_{j}$ the volume source of $q_{j}$. Upon substituting into the first term in the right-hand side of (3) we find

$$
\frac{\partial}{\partial t}\left(V_{j}\left\langle q_{j}\right\rangle\right)=\int_{V_{j}}\left[-\nabla \cdot\left(\mathbf{u}_{j} q_{j}-\boldsymbol{\phi}_{j}\right)+\theta_{j}\right] d^{3} \xi+\oint_{S_{i}} q_{j} \mathbf{v}_{i} \cdot \mathbf{n}_{j} d S_{i},
$$

${ }_{8}$ or, upon using the divergence theorem,

$$
\frac{\partial}{\partial t}\left(V_{j}\left\langle q_{j}\right\rangle\right)+\int_{S_{j}} q_{j} \mathbf{u}_{j} \cdot \mathbf{n}_{j} d S_{j}=\int_{S_{j}} \boldsymbol{\phi}_{j} \cdot \mathbf{n}_{j} d S_{j}+\int_{S_{i}}\left[-q_{j}\left(\mathbf{u}_{j}-\mathbf{v}_{i}\right)+\boldsymbol{\phi}_{j}\right] \cdot \mathbf{n}_{j} d S_{i}+\int_{V_{j}} \theta_{j} d^{3} \xi .
$$

Here we have separated the surface integrals over the interface (in the righthand side) from those over the surface of the averaging volume (in the lefthand side).

Now we use the exact, purely geometric theorem (see e.g. Gray and Lee, 1977; Prosperetti and Tryggvason, 2009)

$$
\int_{S_{j}} \boldsymbol{\phi}_{j} \cdot \mathbf{n}_{j} d S_{j}=\nabla \cdot \int_{V_{j}} \boldsymbol{\phi}_{j} d^{3} \xi=\nabla \cdot\left(V_{j}\left\langle\boldsymbol{\phi}_{j}\right\rangle\right)
$$

54 (actually valid for any vector or higher-order tensor) to rewrite this result as $\frac{\partial}{\partial t}\left(V_{j}\left\langle q_{j}\right\rangle\right)+\nabla \cdot\left(V_{j}\left\langle q_{j} \mathbf{u}_{j}\right\rangle\right)=\nabla \cdot\left(V_{j}\left\langle\boldsymbol{\phi}_{j}\right\rangle\right)+\int_{S_{i}}\left[-q_{j}\left(\mathbf{u}_{j}-\mathbf{v}_{i}\right)+\boldsymbol{\phi}_{j}\right] \cdot \mathbf{n}_{j} d S_{i}+V_{j}\left\langle\theta_{j}\right\rangle$. 
71

Upon division by $V$, assumed to be independent of $\mathbf{x}$ as already stated, and upon introduction of the volume fraction $\alpha_{j}$ of the $j$-phase defined by

$$
\alpha_{j}=\frac{V_{j}}{V},
$$

we find the general averaged balance law

$$
\begin{aligned}
\frac{\partial}{\partial t}\left(\alpha_{j}\left\langle q_{j}\right\rangle\right)+ & \nabla \cdot\left(\alpha_{j}\left\langle q_{j} \mathbf{u}_{j}\right\rangle\right)=\nabla \cdot\left(\alpha_{j}\left\langle\boldsymbol{\phi}_{j}\right\rangle\right) \\
& +\frac{1}{V} \int_{S_{i}}\left[-q_{j}\left(\mathbf{u}_{j}-\mathbf{v}_{i}\right)+\boldsymbol{\phi}_{j}\right] \cdot \mathbf{n}_{j} d S_{i}+\alpha_{j}\left\langle\theta_{j}\right\rangle .
\end{aligned}
$$

\section{Separation of scales}

While (10) is formally exact, its physical transparency can be enhanced as we now show. We focus on the terms containing the non-convective flux $\boldsymbol{\phi}_{j}$ in (8), namely

$$
\boldsymbol{\Phi}_{j} \equiv \boldsymbol{\nabla} \cdot\left(V_{j}\left\langle\boldsymbol{\phi}_{j}\right\rangle\right)+\int_{S_{i}} \boldsymbol{\phi}_{j} \cdot \mathbf{n}_{j} d S_{i} .
$$

We show the development explicitly for $j=1$; the results for $j=2$ can be recovered by simply interchanging the indices 1 and 2 .

Let us define

$$
\overline{\boldsymbol{\phi}}=\alpha_{1}\left\langle\boldsymbol{\phi}_{1}\right\rangle+\alpha_{2}\left\langle\boldsymbol{\phi}_{2}\right\rangle .
$$

By its definition, this quantity captures the large-scale structure of the $\phi$ field and may be expected to be slowly varying over the scale of the averaging volume. Upon multiplying by $V$ (assumed independent of position) and taking the divergence we have

$$
V \nabla \cdot \bar{\phi}=\nabla \cdot\left(V_{1}\left\langle\phi_{1}\right\rangle\right)+\nabla \cdot\left(V_{2}\left\langle\phi_{2}\right\rangle\right) .
$$

This relation permits us to re-write (11) with $j=1$ as

$$
\boldsymbol{\Phi}_{1}=V \nabla \cdot \overline{\boldsymbol{\phi}}-\boldsymbol{\nabla} \cdot\left(V_{2}\left\langle\boldsymbol{\phi}_{2}\right\rangle\right)+\int_{S_{i}} \boldsymbol{\phi}_{1} \cdot \mathbf{n}_{1} d S_{i} .
$$

On the phase interface $S_{i}$ the conservation law of of the generic quantity $q_{j}$ imposes a relation between $\phi_{1}$ and $\phi_{2}$ of the general form

$$
\left(\phi_{1}-\phi_{2}\right) \cdot \mathbf{n}_{1}=\gamma,
$$


Let us now set in this equation

$$
\phi_{2}=\bar{\phi}+\phi_{2}^{\prime}
$$

${ }_{81}$ By the definition of $\bar{\phi}, \phi_{2}^{\prime}$ captures the local small-scale fluctuations of the ${ }_{82}$ field $\phi_{2}$ in the averaging volume. The contribution of $\bar{\phi}$ to the first integral ${ }_{83}$ in (17) is

$$
\oint_{S_{2}+S_{i}} \bar{\phi} \cdot \mathbf{n}_{2} d S=\int_{V_{2}} \nabla \cdot \bar{\phi} d V_{2} .
$$

${ }_{84}$ Since $\bar{\phi}$ is slowly varying over the scale of the averaging volume, we approx85 imate the volume integral as

$$
\oint_{S_{2}+S_{i}} \bar{\phi} \cdot \mathbf{n}_{2} d S \simeq V_{2} \boldsymbol{\nabla} \cdot \bar{\phi},
$$

${ }_{86}$ which is equivalent to assuming that $\boldsymbol{\nabla} \cdot \bar{\phi}$ is essentially constant over the 87 averaging volume $V$ or that, equivalently, $\bar{\phi}$ is adequately represented by a 88 linear function of position inside $V$. Upon substituting into (17) we then find

$$
\boldsymbol{\Phi}_{1} \simeq V \boldsymbol{\nabla} \cdot \overline{\boldsymbol{\phi}}-V_{2} \boldsymbol{\nabla} \cdot \overline{\boldsymbol{\phi}}-\oint_{S_{2}+S_{i}} \boldsymbol{\phi}_{2}^{\prime} \cdot \mathbf{n}_{2} d S+\int_{S_{i}} \gamma d S_{i}
$$

89 i.e.

$$
\boldsymbol{\Phi}_{1} \simeq V_{1} \boldsymbol{\nabla} \cdot \overline{\boldsymbol{\phi}}-\oint_{S_{2}+S_{i}} \boldsymbol{\phi}_{2}^{\prime} \cdot \mathbf{n}_{2} d S+\int_{S_{i}} \gamma d S_{i} .
$$


With this development the general averaged balance law (10) becomes

$$
\begin{gathered}
\frac{\partial}{\partial t}\left(\alpha_{1}\left\langle q_{1}\right\rangle\right)+\nabla \cdot\left(\alpha_{1}\left\langle q_{1} \mathbf{u}_{1}\right\rangle\right)=\alpha_{1} \boldsymbol{\nabla} \cdot \overline{\boldsymbol{\phi}}-\frac{1}{V} \oint_{S_{2}+S_{i}} \boldsymbol{\phi}_{2}^{\prime} \cdot \mathbf{n}_{2} d S \\
+\frac{1}{V} \int_{S_{i}}\left[-q_{1}\left(\mathbf{u}_{1}-\mathbf{v}_{i}\right) \cdot \mathbf{n}_{1}+\gamma\right] d S_{i}+\alpha_{1}\left\langle\theta_{1}\right\rangle,
\end{gathered}
$$

with a similar equation for the other phase. This is the main result of this work. It shows that both phases are acted upon the by same non-convective flux $\bar{\phi}$ apportioned according to the respective volume fractions. The first surface integral in the right-hand side accounts for the local interaction between the phases and can be calculated as if the situation was locally uniform since the large-scale structure of the flow is captured by $\bar{\phi}$.

\section{An example: Disperse flow}

Let us consider the application of the result (23) to the case of disperse flow in which arbitrarily shaped drops or particles (phase 2) are suspended in a continuous phase (phase 1). The same result would apply to bubbles provided they maintain a constant volume as, in the derivation that follows (but not in the general argument of the previous section), we assume the two phases to be incompressible. For simplicity of exposition we use the word "drop" to designate any type of disperse entity.

In this application the interface $S_{i}$ consists of the collection of the surfaces of the drops entirely contained in the averaging volume, plus the surfaces of the incomplete drops only part of which is in the averaging volume (see figure 1).

For momentum balance, for which $q_{j}=\rho_{j} \mathbf{u}_{j}, \boldsymbol{\phi}_{j}=\boldsymbol{\sigma}_{j}$ is the stress tensor for phase $j$. The total force exerted by the continuous phase on each complete drop (i.e., on each drop entirely contained within the averaging volume $V$ ) is given by

$$
\mathbf{f}_{t}=\oint_{s_{d}} \boldsymbol{\sigma}_{1} \cdot \mathbf{n}_{d} d s_{d}=\oint_{s_{d}} \boldsymbol{\sigma}_{2} \cdot \mathbf{n}_{d} d s_{d},
$$

in which $s_{d}$ is the drop surface and $\mathbf{n}_{d}$ is the outwardly directed unit normal. Actually $\boldsymbol{\sigma}_{2} \cdot \mathbf{n}_{d}$ differs from $\boldsymbol{\sigma}_{1} \cdot \mathbf{n}_{d}$ because of the effect of surface tension but, after integration over the entire drop surface, this contribution vanishes as surface tension does not exert any net force on the whole drop (see e.g. 
Prosperetti and Jones, 1984; Hesla et al., 1993). We write

$$
\mathbf{f}_{t}=\oint_{s_{d}} \boldsymbol{\sigma}_{2} \cdot \mathbf{n}_{d} d s_{d}=\oint_{s_{d}}\left(\overline{\boldsymbol{\sigma}}+\boldsymbol{\sigma}_{2}^{\prime}\right) \cdot \mathbf{n}_{d} d s_{d} \simeq v_{d} \boldsymbol{\nabla} \cdot \overline{\boldsymbol{\sigma}}+\oint_{s_{d}} \boldsymbol{\sigma}_{2}^{\prime} \cdot \mathbf{n}_{d} d s_{d},
$$

where $v_{d}$ is the drop volume. Note that the normal $\mathbf{n}_{d}$ has the same direction as the normal $\mathbf{n}_{2}$ appearing in (23). The first term is the force due to the large-scale flow in which the drop is immersed and is akin to a pseudobuoyancy force (see e.g. Jackson, 2000; Prosperetti and Tryggvason, 2009), while the second one is responsible for the force due to the local conditions in the neighborhood of the drop such as drag, lift, added mass and others; we set

$$
\mathbf{f}=\oint_{s_{d}} \boldsymbol{\sigma}_{2}^{\prime} \cdot \mathbf{n}_{d} d s_{d}
$$

The situation of the drops only partially contained in the averaging volume is different as their contribution to $S_{i}$ is not via integration over a closed surface. Thus, for example, the result of the integral analogous to (24) for these drops would depend on the absolute pressure level, which would introduce an unphysical feature in the modelling of incompressible flows. Furthermore, these drops contribute to the integration over $S_{2}$ in (23). The simplest way to deal with this situation probably is to count the contribution of these drops proportionally to the fraction of their volume (or surface area) contained within the averaging volume. With this understanding we find for the second term in the right-hand side of $(23)$

$$
\frac{1}{V} \oint_{S_{2}+S_{i}} \boldsymbol{\sigma}_{2}^{\prime} \cdot \mathbf{n}_{2} d S=\frac{1}{V} \sum_{d} \mathbf{f}_{d}=n \overline{\mathbf{f}}
$$

where the summation is over all the drops in the averaging volume, possibly weighted proportionally to their inclusion in the averaging volume as explained before. In the last step we have introduced the drop number density $n$ and the mean hydrodynamic force acting on the drops $\overline{\mathbf{f}}$.

Assuming the density of the continuous phase to be constant and neglecting mass transfer phenomena across the drop interfaces, (23) becomes then

$$
\begin{gathered}
\rho_{1}\left(\frac{\partial}{\partial t}\left(\alpha_{1}\left\langle\mathbf{u}_{1}\right\rangle\right)+\nabla \cdot\left(\alpha_{1}\left\langle\mathbf{u}_{1} \mathbf{u}_{1}\right\rangle\right)\right)=\alpha_{1} \nabla \cdot \overline{\boldsymbol{\sigma}}-\frac{1}{V} \sum_{d} \mathbf{f}_{d} \\
+\frac{1}{V} \int_{S_{i}} \gamma d S_{i}+\alpha_{1} \rho_{1} \mathbf{g}
\end{gathered}
$$


where $\gamma$ is the (vector) contribution of the surface forces and the volume source of momentum per unit mass has been written as $\mathbf{g}$. The mean stress $\bar{\sigma}$ may be decomposed into pressure and viscous components and closed, e.g., as

$$
\bar{\sigma}_{i j}=-\bar{p} \delta_{i j}+\bar{\tau}_{i j}=-\bar{p} \delta_{i j}+\mu_{e f f}\left(\frac{\partial \bar{u}_{i}}{\partial x_{j}}+\frac{\partial \bar{u}_{j}}{\partial x_{i}}\right),
$$

with $\mu_{\text {eff }}$ an effective shear viscosity and $\overline{\mathbf{u}}=\alpha_{1}\left\langle\mathbf{u}_{1}\right\rangle+\alpha_{2}\left\langle\mathbf{u}_{2}\right\rangle$ as in (12). A diagonal term proportional to $\boldsymbol{\nabla} \cdot \overline{\mathbf{u}}$ is absent since the mixture is incompressible. With these choices (28) becomes

$$
\begin{gathered}
\rho_{1}\left(\frac{\partial}{\partial t}\left(\alpha_{1}\left\langle\mathbf{u}_{1}\right\rangle\right)+\boldsymbol{\nabla} \cdot\left(\alpha_{1}\left\langle\mathbf{u}_{1} \mathbf{u}_{1}\right\rangle\right)\right)=-\alpha_{1} \boldsymbol{\nabla} \bar{p}+\alpha_{1} \boldsymbol{\nabla} \cdot \overline{\boldsymbol{\tau}}-\frac{1}{V} \sum_{d} \mathbf{f}_{d} \\
+\frac{1}{V} \int_{S_{i}} \gamma d S_{i}+\alpha_{1} \rho_{1} \mathbf{g} .
\end{gathered}
$$

The integral over $S_{i}$ is obviously difficult to model. If the area fraction occupied by the drops on the surface of the averaging volume is $\alpha_{s}$ we have, in order of magnitude,

$$
\left|\frac{1}{V} \int_{S_{i}} \gamma d S_{i}\right| \sim \frac{\alpha_{s} S}{V} \frac{2 \gamma}{r_{d}}
$$

where $S$ is the surface of the averaging volume, $\gamma$ the surface tension coefficient and $r_{d}$ the drop radius. The hydrodynamic force is very often dominated by drag so that, again in order of magnitude,

$$
n|\overline{\mathbf{f}}| \sim n \mu_{1} r_{d} w_{d},
$$

with $w_{d}$ the magnitude of the drop-fluid relative velocity and $\mu_{1}$ the viscosity of the continuous phase. The ratio of these two terms is therefore

$$
\frac{2\left(\alpha_{s} S / V\right) \gamma / r_{d}}{n \mu_{1} r_{d} w_{d}}=2 \frac{\alpha_{s} S / r_{d}^{2}}{n V} \frac{\gamma}{\mu_{1} w_{d}}=\frac{N_{S}}{N_{V}} \frac{2}{C a},
$$

in which $N_{S}$ and $N_{V}$ are the numbers of drops on the surface of, and inside, the averaging volume, respectively, and $C a=\mu_{1} w_{d} / \gamma$ is a representative capillary number. One would typically expect $N_{S}<N_{V}$. Thus, provided the capillary number is not too small, the surface integral in (28) and (30) may be neglected. 
177

The disperse phase can be treated in the same way except that, in the final result, one can take a step similar to the second one in (27) thus finding a momentum equation for the "average drop" contained in the averaging volume, which it is convenient to write in the Lagrangian form:

$$
\frac{d}{d t}\left(\overline{m \mathbf{u}_{d}}\right)=\bar{v}_{d} \boldsymbol{\nabla} \cdot \overline{\boldsymbol{\sigma}}+\overline{\mathbf{f}}+\bar{m} \mathbf{g},
$$

where $m$ and $\mathbf{u}_{d}$ are the drop mass and velocity. Surface tension causes deformation of the drop but no translational motion, as noted before, and, accordingly, it has been omitted. An alternative to the use of (34) is to write the equation for each individual drop, thus adopting a Lagrangian description as is done, for example, in the point-particle or discrete-element models (see e.g. Ferrante and Elghobashi, 2003; Eaton, 2009; Deen et al., 2007).

The thermal energy balance can be treated in a similar way. For an incompressible continuous phase with constant properties we would have $q_{1}=$ $\rho_{1} c_{p 1} T_{1}$, with $c_{p 1}$ the constant-pressure specific heat and $T_{1}$ the temperature, while $\phi_{1}=-\mathbf{q}_{1}$ the heat flux. In this case the analog of (25) is

$$
\mathbf{Q}_{t}=\oint_{s_{d}}\left(-\overline{\mathbf{q}}-\mathbf{q}_{2}^{\prime}\right) \cdot \mathbf{n}_{d} d s_{d} \simeq-v_{d} \boldsymbol{\nabla} \cdot \overline{\mathbf{q}}-\oint_{s_{d}} \mathbf{q}_{2}^{\prime} \cdot \mathbf{n}_{d} d s_{d},
$$

and, upon letting

$$
\mathbf{Q}=-\oint_{s_{d}} \mathbf{q}_{2}^{\prime} \cdot \mathbf{n}_{d} d s_{d},
$$

we find the energy balance in the form

$$
\rho_{1} c_{p 1}\left(\frac{\partial}{\partial t}\left(\alpha_{1}\left\langle T_{1}\right\rangle\right)+\nabla \cdot\left(\alpha_{1}\left\langle T_{1} \mathbf{u}_{1}\right\rangle\right)\right)=-\alpha_{1} \boldsymbol{\nabla} \cdot \overline{\mathbf{q}}-n \overline{\mathbf{Q}} .
$$

Here we have neglected volume sources of internal energy such as viscous dissipation. Furthermore, because of the neglect of phase change at the drop surface, the right-hand side of the interface balance (15) vanishes.

The average heat flux can be closed as before as

$$
\overline{\mathbf{q}}=-k_{e f f} \nabla \bar{T},
$$

with $k_{\text {eff }}$ an effective conductivity and $\bar{T}=\alpha_{1}\left\langle T_{1}\right\rangle+\alpha_{2}\left\langle T_{2}\right\rangle$ as in (12). The internal energy balance for the drops can be readily written down as before:

$$
\frac{d}{d t}\left(c_{p 1} \overline{m T_{d}}\right)=-\bar{v}_{d} \boldsymbol{\nabla} \cdot \overline{\mathbf{q}}+\overline{\mathbf{Q}} .
$$


Similarly to the use of a drag coefficient in the closure of the interphase force, a heat transfer coefficient $h$ can be used to close $\mathbf{Q}$ :

$$
\mathrm{Q}=h s_{d}\left(\left\langle T_{1}\right\rangle-T_{d}\right)
$$

\section{Discussion}

The result (23) shows two significant features concerning the contribution of the non-convective flux $\phi$ to the general averaged balance law. The first one is the the appearance of the flux averaged over the entire mixture rather than over the individual phases. The second one is the presence of the volume fraction outside, rather than inside, the divergence operator.

In an earlier paper (Zhang and Prosperetti, 1997) we have derived the momentum and energy equations in a dilute fluid-particle flow by means of ensemble averaging. The results conform precisely with (28) and (37), respectively, with $\overline{\boldsymbol{\sigma}}$ and $\overline{\mathbf{q}}$ as in (29) and (38). The very different method of derivation lends considerable support to the validity of these results.

It is well appreciated in the literature that, in the pressure term of the momentum equation, the volume fraction must be outside the gradient operator as in (30) in order to avoid unphysical results, e.g. in the case of a spatially non-uniform system on which no forces are acting (see e.g. Jackson, 2000; Prosperetti and Tryggvason, 2009). However, the viscous term is often written as $\boldsymbol{\nabla} \cdot\left(\alpha_{1}\left\langle\boldsymbol{\tau}_{1}\right\rangle\right)$. While this form agrees with the result of straight volume averaging as in the first term in the right-hand side of (10), the problem remains of closing $\left\langle\boldsymbol{\tau}_{1}\right\rangle$ as well as the interface integral in (10). The first objective is often accomplished by simply setting

$$
\left\langle\boldsymbol{\tau}_{1, i j}\right\rangle=\hat{\mu}_{1}\left(\frac{\partial}{\partial x_{j}}\left\langle u_{1, i}\right\rangle+\frac{\partial}{\partial x_{i}}\left\langle u_{1, j}\right\rangle-\frac{2}{3} \boldsymbol{\nabla} \cdot\left\langle\mathbf{u}_{1}\right\rangle \delta_{i j}\right),
$$

with $\hat{\mu}_{1}$ a possibly modified fluid viscosity. This is the choice made by a large number of investigators; representative examples of what could be a very long list are the model employed in the code MFIX (Benyahia et al., 2012) as well as the studies by Deen et al. (2007), Janet et al. (2015) and many others.

In a recent paper, Capecelatro and Desjardins (2013) derive a momentum equation for the continuous phase by a mollified form of volume averaging separating terms slowly and rapidly varying in space. Upon combining 
their equations (26), (27), (40) and (41) one finds that (upon omitting nonessential features) the contribution of the stress terms in the right-hand side of the fluid momentum equation can be written as

$$
-\alpha_{1} \nabla \bar{p}+\alpha_{1} \boldsymbol{\nabla} \cdot\left[\mu_{e f f}\left(\nabla \tilde{\mathbf{u}}_{1}+\nabla \tilde{\mathbf{u}}_{1}^{T}-\frac{2}{3} \boldsymbol{\nabla} \cdot \tilde{\mathbf{u}}_{1}\right)\right]-\frac{1}{V_{c}} \sum_{i} \mathbf{f}_{i},
$$

with the sum extended to the particles in the computational cell of volume $V_{c}$ and $\mathbf{f}$ the hydrodynamic force on each particle. Here $\tilde{\mathbf{u}}_{1}$ is defined as "the volume-filtered [i.e., slowly varying] fluid velocity" and is therefore similar, although not exactly equal, to the slowly varying volumetric flux of the whole mixture $\overline{\mathbf{u}}$ introduced before in (29); the last term is the same as (27). The derivation by Capecelatro and Desjardins (2013) confirms the presence of the volume fraction outside the divergence operator in spite of the (probably quantitatively) minor difference between $\tilde{\mathbf{u}}_{1}$ and $\overline{\mathbf{u}}$.

The derivation of section 3 shows that the proper position of the volume fraction is outside the gradient operator not only for the momentum equation, but for all the balance laws. This is at variance with most of the models in current use (see e.g. Janet et al., 2015; Patil et al., 2015, for some recent examples).

\section{Conclusions}

By taking advantage of the separation of scales between macroscopic and local microscopic fields, we have recast the mathematical form in which nonconvective fluxes $\boldsymbol{\phi}_{j}$ (e.g. stress, heat flux and others) appear in the volumeaveraged equation of the $j$-th phase. We have shown that, in place of fields $\left\langle\boldsymbol{\phi}_{j}\right\rangle$ averaged over the individual phases, it is preferable to use a single, globally averaged field $\bar{\phi}=\alpha_{1}\left\langle\phi_{1}\right\rangle+\alpha_{2}\left\langle\phi_{2}\right\rangle$, the action of which is apportioned according to the volume fraction of each phase as shown in (23). This step has several advantages.

In the first place, it provides a clear guidance as to how to interpret and use the terms containing $\boldsymbol{\phi}_{j}$ in the unclosed form (10) of the general volume-averaged balance equation usually encountered in the literature.

Secondly, the derivation shows the much greater significance of the mixture flux compared to that of the individual phases. When the normal component of $\boldsymbol{\phi}_{j}$ is continuous across the interface, while $\left\langle\nabla \cdot \boldsymbol{\phi}_{j}\right\rangle \neq \boldsymbol{\nabla} \cdot\left\langle\boldsymbol{\phi}_{j}\right\rangle$ as differentiation and averaging do not commute, it can be shown that it is 
nonetheless true that $\alpha_{1}\left\langle\boldsymbol{\nabla} \cdot \boldsymbol{\phi}_{1}\right\rangle+\alpha_{2}\left\langle\boldsymbol{\nabla} \cdot \boldsymbol{\phi}_{2}\right\rangle=\boldsymbol{\nabla} \cdot\left(\alpha_{1}\left\langle\boldsymbol{\phi}_{1}\right\rangle+\alpha_{2}\left\langle\boldsymbol{\phi}_{2}\right\rangle\right) \equiv \boldsymbol{\nabla} \cdot \overline{\boldsymbol{\phi}}$. Similarly, if some quantity such as the temperature $T_{j}$ is continuous across the interface, it is true that $\alpha_{1}\left\langle\nabla T_{1}\right\rangle+\alpha_{2}\left\langle\nabla T_{2}\right\rangle=\nabla\left(\alpha_{1}\left\langle T_{1}\right\rangle+\alpha_{2}\left\langle T_{2}\right\rangle\right) \equiv \nabla \bar{T}$. In the case of heat conduction, for example, this circumstance justifies the introduction of an effective thermal conductivity $k_{\text {eff }}$ of the mixture defined so that the mixture heat flux $\overline{\mathbf{q}}$ is closed as $\overline{\mathbf{q}}=-k_{e f f} \boldsymbol{\nabla} \bar{T}$ as done before in (38) (see e.g. Sangani and Acrivos, 1983; Chang and Acrivos, 1986; Bonnecaze and Brady, 1990; Marchioro and Prosperetti, 1999). A similar relation permits the introduction of an effective viscosity in a mean stressvolumetric flux closure relation (see e.g. Batchelor, 1970, 1974; Nunan and Keller, 1984). There is therefore a direct connection between the voluminous literature on effective properties of disperse systems (see e.g. Torquato, 2011) and the volume averaged equations.

Thirdly, the derivation clarifies the proper position of the volume fractions in the terms involving the divergence of the non-convective fluxes.

Our result is general and only rests on the hypothesis that the spatial variation of the combined average flux $\bar{\phi}$ is adequately represented by a linear function of position within the averaging volume, which seems a rather mild assumption whenever the use of volume averaging is useful. No further hypotheses on the nature of the flow (e.g., about specific flow regimes) are necessary.

Finally, it is to be noted that the present results coincide with earlier ones obtained by the completely different method of ensemble averaging for the special case of a dilute suspension of spherical particles in a fluid (Zhang and Prosperetti, 1997). This coincidence, together with the difference between the methods of derivation, lends a strong support to their validity.

\section{Acknowledgments}

AP has been supported by the U.S. National Science Foundation under grant CBET 1335965. SC's participation was made possible by a grant from The Gulf of Mexico Research Initiative. The funders had no role in study design, data collection and analysis, decision to publish, or preparation of the manuscript.

\section{References}

Batchelor, G., 1970. The stress system in a suspension of force-free particles. J. Fluid Mech. 41, 545-570. 
Batchelor, G. K., 1974. Transport properties of two-phase materials with random structure. Annu. Rev. Fluid Mech. 6, 227-254.

Benyahia, S., Syamlal, M., O'Brien, T. J., 2012. Summary of MFIX Equations 2012-1. DOE National Energy Technology Laboratory, https://mfix.nets.doe.gov/documentation/MFIXEquations2012-1.pdf.

Bonnecaze, R., Brady, J., 1990. A method for determining the effective conductivity of dispersions of particles. Proc. Roy. Soc. Lond. A430, 285-317.

Capecelatro, J., Desjardins, O., 2013. An Euler-Lagrange strategy for simulating particle-laden flows. J. Comput. Phys. 238, 1-31.

Chang, E., Acrivos, A., 1986. Rate of heat conduction from a heated sphere to a matrix containing passive spheres of a different conductivity. J. Appl. Phys. 59, 3375-3382.

Deen, N., van Sint Annaland, M., van der Hoef, M., Kuipers, J. A. M., 2007. Review of discrete particle modeling of fluidized beds. Chem. Eng. Sci. 62, $28-44$.

Eaton, J. K., 2009. Two-way coupled turbulence simulations of gas-particle flows using point-particle tracking. Int. J. Multiphase Flow 35, 792-800.

Ferrante, A., Elghobashi, S., 2003. On the physical mechanisms of two-way coupling in particle-laden isotropic turbulence. Phys. Fluids 15, 315-329.

Gray, W. G., Lee, P. C., 1977. On the theorem for local volume averaging for multiphase systems. Int. J. Multiphase Flow 3, 333-340.

Hesla, T. I., Huang, A. Y., Joseph, D. D., 1993. A note on the net force and moment on a drop due to surface forces. J. Colloid Interface Sci. 158, $255-257$.

Ishii, M., Ibiki, T., 2011. Thermo-Fluid Dynamics of Two-Phase Flow. Springer, New York NY.

Jackson, R., 2000. The Dynamics of Fluidized Particles. Cambridge UP, Cambridge UK. 
Janet, J. P., Liao, Y., Lucas, D., 2015. Heterogeneous nucleation in CFD simulation of flashing flows in converging-diverging nozzles. Int. J. Multiphase Flow 74, 106-17.

Marchioro, M., Prosperetti, A., 1999. Conduction in non-uniform composites. Proc. R. Soc. London A455, 1483-1508.

Nigmatulin, R. I., Lahey, R. T., Drew, D. A., 1996. On the different forms of momentum equations and on the intra- and interphase interaction in the hydromechanics of a monodisperse mixture. Chem. Eng. Comm. 141-142, 287-302.

Nunan, K., Keller, J., 1984. Effective viscosity of a periodic suspension. J. Fluid Mech. 142, 269-287.

Patil, A. V., Peters, E. A. J. F., Kuipers, J. A. M., 2015. Comparison of CFD-DEM heat transfer simulations with infrared/visual measurements. Chem. Eng. J. 277, 388-401.

Prosperetti, A., Jones, A., 1984. Pressure forces in dispersed two-phase flows. Int. J. Multiphase Flow 10, 425-440.

Prosperetti, A., Tryggvason, G., 2009. Computational Methods in Multiphase Flow, paperback Edition. Cambridge University Press, Cambridge UK.

Sangani, A., Acrivos, A., 1983. The effective conductivity of a periodic array of spheres. Proc. Roy. Soc. Lond. A386, 263-275.

Torquato, S., 2011. Random Homogeneous Materials: Macroscopic Properties, 2nd Edition. Springer, New York.

Whitaker, S., 1999. The Method of Volume Averaging. Springer, New York NY.

Zhang, D. Z., Prosperetti, A., 1997. Momentum and energy equations for disperse two-phase flows and their closure for dilute suspensions. Int. J. Multiphase Flow 23, 425-453. 


\section{Figure captions}

338 Figure 1: Averaging volume in a two-phase system. The surface of the 339 averaging volume, $S=S_{1}+S_{2}$, consists of a portion $S_{1}$ in contact with phase ${ }_{340} 1$ (continuous thick line) and a portion $S_{2}$ in contact with phase 2 (dashed 341 thick line). Inside the averaging volume the two phases are separated by an 342 interface $S_{i}$ (thin lines) which may consist of disjoint parts as in this figure.

343 Figure 2: The unit normals are defined to be directed out of the correspond344 ing phase. 